\title{
Decision-making for Carotid Endarterectomy. The Trials are Only the Start
}

Can. J. Neurol. Sci. 2002; 29: 302-304

Ancient civilizations created gods and symbols to focus on their needs and fears. Janus was created to watch over Roman portals and had two faces to allow him to look simultaneously in two directions. Centuries later but prior to European intrusion, African artists not uncommonly gave two or even three faces to their ritual masks (Figure). When the various societies of biostatisticians create their particular god(ess) it would be wise of them to consider a two-faced image to symbolize the imperative required in the interpretation of even the most impressive study: evidence of efficacy will only carry great weight at the bedside if it can practically be applied. Does the efficacy convert to effectiveness (is the therapy efficacious)? Is the risk imposed by a new drug clearly overwhelmed by its singular benefit? Will the requisite technology be available sufficiently widely and reliably used to match the sophisticated technology upon which results were obtained? In the case of surgical procedures, will the skill of surgeons outside a trial be sufficient to match the skill of the surgeons selected for their

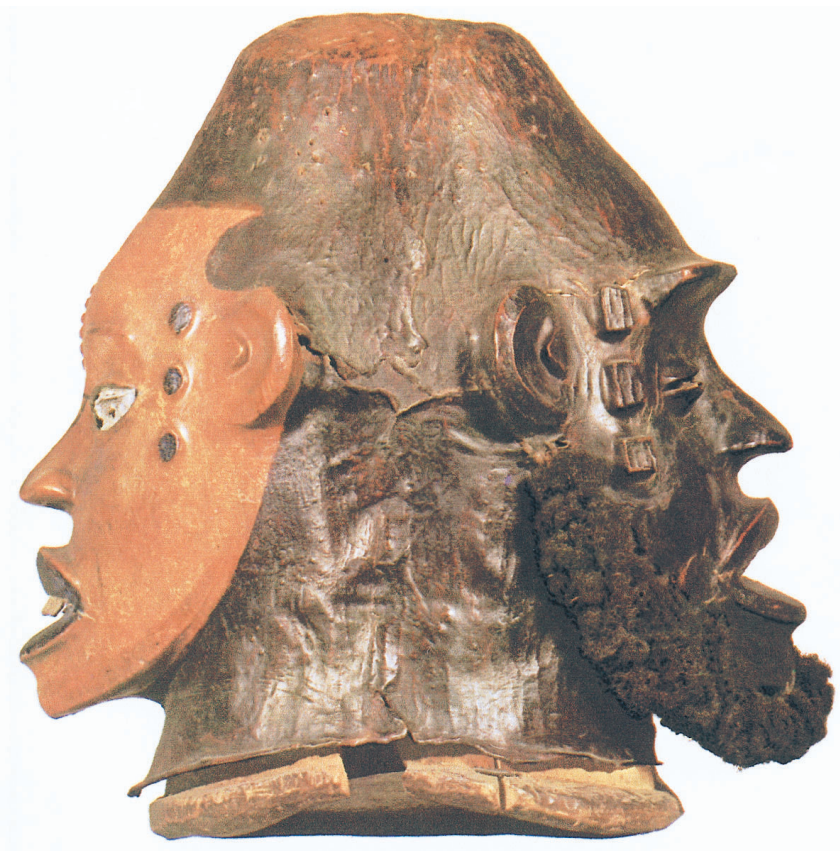

Figure: Two faces of a ritual mask from the Ekoi tribe of the Cross River area of Nigeria. The male face with eyes shut reflects on the past, simultaneously with female face looks ahead. Civilizations since Roman times have created sprits for gods like Janus, symbolizing the need to look at the same time in more than a single direction.

Copyright The British Museum. Courtesy and thanks to Dr J.C.E. Kaufmann. expertise to carry out the procedure with minimal complications? Focusing specifically upon carotid endarterectomy (CE) can the published results be applied generically to the gamut of patients afflicted with carotid stenosis or are there specific groups identifiable who will benefit most and perhaps some who will even be harmed?

In 1991 when the North American Symptomatic Carotid Endarterectomy Trial (NASCET) published the results favoring $\mathrm{CE}$ for the subgroup of patients with symptoms due to severe stenosis and seven years later for those with moderate stenosis, the only analyses were for grades of benefit relating to patients with the variable degree of stenosis above and below $70 \% .^{1,2}$ Subgroups whose base-line characteristics might give reason to believe that they would receive particularly good benefit were not examined at once. Nor were groups that might be better off with only medical care. As our team of tolerant investigators knew very well, NASCET required extensive documentation so that a fulsome database could later be tapped to explore the impact of a variety of variables.

The data for most of these specifically-defined groups of patients have subsequently been reported individually in a series of papers. A recent review has summarized the findings. ${ }^{3}$ Not unexpectedly, the patients at highest risk if treated medically, gained most from surgical treatment and conversely patients at the lowest medical risk gained the least. For example: males, patients without good collateral circulation, with hemisphere as compared to retinal symptoms, with stroke rather than TIA and with CT evidence of infarction were at highest risk on conservative therapy and had the best stroke-free survival after CE. It came as a surprise to the investigators that in two other types of patients with high medical risk there was exceptionally good benefit from CE: the elderly ( $>75$ years) and those with tandem intracranial stenosis. The main results and the secondary analyses in NASCET must be read recalling that the perioperative complication rate for stroke and death was $5.8 \%$ with severe and $6.5 \%$ with moderate stenosis. Above these figures the benefit narrows and soon disappears.

The second important caveat is that patients with recent evidence of threatening heart disease and evidence of failure of other vital organs were specifically excluded from the trial. Nobody knows what benefit or harm will result if they are included. Lesser benefit was found in patients at low medical risk. The most striking example was the muted benefit found in all patients with $<70 \%$ stenosis. At this level of stenosis men, the elderly, those without good collateral, those presenting with hemisphere events, and those with tandem neck and intracranial stenosis are among those who should be encouraged to have CE. Their opposites (including women and younger patients) cannot 
be presented with convincing evidence that they are candidates. In conjunction with moderate stenosis and a low vascular risk profile, there are recognizable patients with moderate stenosis who will not only fail to benefit but, as a group, may actually be harmed from CE. This would include women and would be applicable to patients of either gender who present with the singular symptom of only-ever amaurosis fugax. ${ }^{4,5}$ Once again, though, if patients in either of these two subgroups with moderate stenosis have a high risk-profile, they are potential candidates.

Confronted with a patient with focal symptoms attributable to a proven carotid arteriosclerotic stenosis, the practitioner must consider all variables in the patient's base-line characteristics, consider what we now know about the risk facing this particular patient treated medically and what has been shown to be feasible or not by the use of CE. The critical point has now been reached where the practitioner must adopt the Janus' attitude: look back at the firm data about overall efficacy from the trials, then scan ahead for specific efficacy for the individual patient. Equally important is to know, with accuracy, the results from the procedure in all communities where it is being utilized. Are the individual patients truly potential candidates? Will the skill of the operating surgeon and the skill of those charged with the postoperative care give the patient the results that will more than match the known risk to be expected from medical care alone in his or her particular instance?

Two studies are published in this issue of the Journal bearing directly upon the commonly-posed question of the applicability of $\mathrm{CE}$ in the community. ${ }^{6,7}$ Both reports touch upon the question of $\mathrm{CE}$ in asymptomatic subjects so that a preliminary brief commentary on the state of knowledge here is in order. Only one of four randomized trials testing for benefit in asymptomatic subjects was able to show a narrow benefit favoring CE. Even then there were distinct problems in applying trial results to the hundreds of thousands of individuals in North America affected with the lesions studied in the Asymptomatic Carotid Arteriosclerosis Study (ACAS). The surgical benefit was sufficiently close to the outlook for patients treated medically that 83 patients need $\mathrm{CE}$ to prevent one stroke in two years (this contrasts with a number needed to treat for elderly symptomatic patients with severe stenosis of 3 ); the perioperative complication rate to achieve this benefit, combined with the trial's angiographic complication rate was extremely low at $2.6 \%$; power of the study, because of the low risk of stroke of $2.4 \%$ in a year facing the medically treated subjects, was the reason offered for not being able to find benefit in women, or in reducing the risk of disabling stroke, or in detecting increasing benefit in those with higher degrees of stenosis. ${ }^{8}$

Any suggestion that the best candidates for CE in asymptomatic subjects are those with highest degrees of stenosis is an extrapolation of analyses done in NASCET and the other symptomatic trials. It may be true but is not based on data that were scientifically obtained. Taking into account the outlook for subjects in the medical arms of the asymptomatic randomized trials and several careful long-term prospective observational studies, it can be stated that benefit for asymptomatic subjects demands a perioperative rate of stroke and death no higher than $3 \%$. A hard look at what is being accomplished in achieving this figure yields discouraging findings. In the Aspirin in Carotid
Surgery Trial (ACE), the complication rate for stroke and death was $4.5 \%$ in 1,215 never-symptomatic subjects operated upon by NASCET surgeons. ${ }^{9,10}$ It did not improve in the 350 subjects treated by surgeons also in the ACAS trial. In a hospital chart review of Medicare patients from 10 states, 7,604 subjects of 10,030 in total submitted to endarterectomy, either had no symptoms or only "non-specific" symptoms and probably were all asymptomatic from the carotid lesion. ${ }^{11}$ In six of the 10 states the procedure was more often harmful than beneficial. There were more strokes than would have been anticipated without the procedure.

Findlay et $\mathrm{al}^{6}$ have admirably confirmed that a process of audit and education will prevent the abuse of carotid endarterectomy. The strategy they describe will be important in ensuring the application of sufficient surgical skill to provide the highest benefit free from immediate and likely long-term occurrence of stroke related to a severely stenosed artery. Unlike many reports in the recent literature, there was careful prospective and independent evaluation of the outcomes of stroke. All patients brought to CE were included. The proportion with the varying degrees of stenosis was reported, as was the presence or absence of symptoms. Because the Edmonton hospitals are the referral centers for every potential patient from a large geographical area, something close to the ideal environment for the pursuit of CE has been achieved. Over four years, the highest perioperative complication rate was $5.2 \%$. This figure related to an average of about 250 procedures for each time-frame. An annual average of 100 procedures was done in asymptomatic subjects and, over the years, the complication rate came out on both sides of the beneficial/harmful dividing line of $3 \%$. Even in a center clearly of exceptional excellence, they correctly regard the use of CE for these asymptomatic subjects as an "uncertain indication". Up until the year 2000 they elected also to allocate all symptomatic patients with moderate stenosis to this "uncertain indication" category. Subsequent scrutiny of the higher-risk patients from the NASCET data-base would allow selected individuals in this moderate range of stenosis to be moved to Findlay's permissive category of "appropriate indication". For all patients in NASCET's moderate range of stenosisis, the number needed to treat was 19 patients. For patients in this range in the European Carotid Surgery Trial, a higher perioperative rate of $9.6 \%$ yielded no surgical benefit. ${ }^{12,13}$

Some confirmation, but also some contrasts, to the optimum surgical record and the enviable tertiary type of referral pattern found in Findlay's geographical area is provided by the Feasby enquiry. ${ }^{7}$ This new survey examines all Canadian CE activity involving a total of 14,268 patients over a three-year period covering the middle years of the other study. The administrative data-base from which the results were compiled allowed the author to glean information on more than 20 baseline characteristics.

Mirabile dictu this data-base in the Canadian Institute for Health Information, like its federal and state counterparts in the United States cannot provide two prognostic items of immense importance: either the presence of symptoms or not, and the degree of stenosis. Without these key items, we are nevertheless able to note that the perioperative mortality is remaining at reasonable levels and that with two provincial exceptions, the stroke morbidity due to $\mathrm{CE}$ is at low levels. Unhappily, the 
suspicion is strong that the stroke morbidity rate given as $3.4 \%$ is more optimistic than the rate would be if the author had been given the opportunity to confirm the absence of any persisting ischemic signs by chart scrutiny or, more accurately, able to report on a careful neurological examination on every patient at discharge.

The 10-state Medicare survey referred to above, included a review of all charts to add some data but did not include impartial examination of patients. It reported a figure of $5.2 \%$ stroke and death complications combining symptomatic and asymptomatic individuals. The figure varied from $4.1 \%$ to $7.7 \%$ in different states. Why are the figures from Feasby and the report based on Medicare less than reported from the NASCET or from the Findlay survey? The answer probably is to be found in a published comparative study of reported complications of $\mathrm{CE}$ : when single surgeons reported on their own operative series, they claimed a complication rate only one-third that reported by neurologists. ${ }^{14}$ To take the complication rates from hospital discharge summaries provides a guideline for trends but not an assurance that efficacy shown in controlled trials has become converted in the outside world to efficaciousness.

Feasby is bold to conclude that regionalization should be considered for some services that demand special skill and experience. For this he is to be congratulated. Vested interests may oppose it but Findlay has shown how effective regionalization and professional education concerning the demands surrounding CE can improve the quality of care in a given geographical area. To this process should be added the need for education of potential patients. A sophisticated public should have critical but reasonable expectations about the quality of care that they can anticipate when they have known carotid stenosis. The profession and the public should be made aware of the good result to be expected under ideal circumstances when appropriate focal symptoms have occurred in conjunction with an appropriate lesion and the requisite surgical skill is at hand. Referring physicians and the public need to be aware of the serious uncertainties surrounding the use of CE in asymptomatic subjects, particularly the widespread failure to match the medical risks facing these individuals with a sufficiently low surgical risk. Health care costs are too high to perpetuate the unbridled use of procedures that do harm. There is no better example in current stroke neurology of the need to look back at the published trial claims and ahead to the reported record of their application. Janus and his African counterparts were wise gods.

H.J.M. Barnett King City, $O N$

\section{REFERENCES}

1. North American Symptomatic Carotid Endarterectomy Trial Collaborators. Beneficial effect of carotid endarterectomy in symptomatic patients with high-grade stenosis. N Engl J Med 1991;325:445-453.

2. Barnett HJM, Taylor DW, Eliasziw M, et al, for the North American Symptomatic Carotid Endarterectomy Trial Collaborators. Benefit of carotid endarterectomy in symptomatic patients with moderate and severe stenosis. N Engl J Med 1998;339:14151425.

3. Barnett HJM, Meldrum HE, Eliasziw M. The appropriate use of carotid endarterectomy. CMAJ 2002;166:1169-1179.

4. Alamowitch S, Eliasziw M, Taylor DW, et al, for the North American Symptomatic Carotid Endarterectomy Trial (NASCET), and the ASA and Carotid Endarterectomy (ACE) Trial Collaborators. The risk and benefit of endarterectomy in women with symptomatic internal carotid artery disease. 2002;(In Press).

5. Benavente O, Eliasziw M, Streifler JY, et al, for the North American Symptomatic Carotid Endarterectomy Trial (NASCET) Collaborators. Prognosis after transient monocular blindness associated with carotid artery stenosis. N Engl J Med 2001;345:1084-1090.

6. Findlay JM, Nykolyn L, Lubkey TB, et al. Auditing carotid endarterectomy: a regional experience. Can J Neurol Sci 2002;29: 326-332.

7. Feasby TE, Quan H, Ghali WA. Provincial carotid endarterectomy outcomes. Can J Neurol Sci 2002;29: 333-336.

8. Executive Committee for the Asymptomatic Carotid Atherosclerosis Study. Endarterectomy for asymptomatic carotid artery stenosis. JAMA 1995:273:1421-1428.

9. Taylor DW, Barnett HJM, Haynes RB, et al, for the ASA and Carotid Endarterectomy (ACE) Trial Collaborators. Low-dose and high-dose acetylsalicylic acid for patients undergoing carotid endarterectomy: a randomized controlled trial. Lancet 1999;353:2179-2184.

10. Inzitari D, Eliasziw M, Gates P, et al, for the North American Symptomatic Carotid Endarterectomy Trial (NASCET) Group. The causes and risk of stroke in subjects with an asymptomatic internal carotid artery. N Engl J Med 2000;342:1693-1700.

11. Kresowik TF, Bratzler D, Karp HR, et al. Multistate utilization, processes, and outcomes of carotid endarterectomy. J Vasc Surg 2001;33:227-235.

12. Barnett HJM, Eliasziw M, Meldrum HE. The prevention of ischemic stroke. BMJ 1999;318:1539-1543

13. Barnett HJM, Eliasziw M, Meldrum HE. Correction to BMJ 1999; 318: 1539-1543. Prevention of ischemic stroke. BMJ 1999;319: 626.

14. Rothwell PM, Slattery W, Warlow CP. A systematic review of the risks of stroke and death due to endarterectomy for symptomatic carotid stenosis. Stroke 1996;27:260-265. 